\title{
AUTOMATIC BUILDING CHANGE DETECTION USING MULTI-TEMPORAL AIRBORNE LiDAR DATA
}

\author{
R. C. dos Santos ${ }^{1 *}$, M. Galo ${ }^{2}$, A. C. Carrilho ${ }^{1}$, G. G. Pessoa ${ }^{1}$, R. A. R. de Oliveira ${ }^{1}$ \\ ${ }^{1}$ São Paulo State University - UNESP, Graduate Program in Cartographic Sciences, \\ Presidente Prudente, São Paulo, Brazil - renato.cesar@unesp.br, andre.carrilho@unesp.br, guilherme.g.pessoa@unesp.br, \\ renanamerico@gmail.com \\ ${ }^{2}$ São Paulo State University - UNESP, Dept. of Cartography, \\ Presidente Prudente, São Paulo, Brazil - mauricio.galo@unesp.br
}

KEY WORDS: Building change detection, Airborne LiDAR data, Shannon entropy

\begin{abstract}
:
The automatic detection of building changes is an essential process for urban area monitoring, urban planning, and database update. In this context, 3D information derived from multi-temporal airborne LiDAR scanning is one effective alternative. Despite several works in the literature, the separation of change areas in building and non-building remains a challenge. In this sense, it is proposed a new method for building change detection, having as the main contribution the use of height entropy concept to identify the building change areas. The experiments were performed considering multi-temporal airborne LiDAR data from 2012 and 2014, both with average density around 5 points $/ \mathrm{m}^{2}$. Qualitative and quantitative analyses indicate that the proposed method is robust in building change detection, having the potential to identify small changes (larger than $20 \mathrm{~m}^{2}$ ). In general, the change detection method presented average completeness and correctness around $97 \%$ and $71 \%$, respectively.
\end{abstract}

\section{INTRODUCTION}

Building change detection is an important process in several applications such as land management, decision making, illegal building identification, disaster management, geographic information database updating, urban growth planning and monitoring. These applications aim at the sustainable and organized development of cities and its citizens, being directly linked to the 2030 agenda for sustainable development (United Nations, 2019).

According to Matikainen et al. (2010), change detection can be performed by means of visual interpretation and manual digitizing. However, it is a time-consuming task, especially in large or continuously changing areas (Manno-Kovacs and Sziranyi, 2015), since the human operators need to search and detect the changed objects by visual inspection over digital images, for instance. In this sense, the development of automated techniques to detect and extract building changes has a strong appeal to both the private and public communities. Besides, such automated techniques can help to save time and money, allowing a recurring database updating.

In the last years, the scientific community has made efforts to develop automatic techniques using remote sensing data. In general, the building change detection can be performed in the 2D space using aerial or satellite images, or in the $3 \mathrm{D}$ space using point clouds generated from photogrammetric techniques or airborne LiDAR scanning systems. The detection process using images usually considers the spectral response, being the final results influenced by several factors: shadows, occlusions, seasonal variations, and different weather conditions. Besides, the use of 2D imagery does not allow the extraction of volumetric change information, unless some digital image matching process is considered. However, the matching process can be affected by occlusion, lack of texture and/or repetitive patterns, for instance. In this sense, LiDAR data emerges as a good alternative, since the $3 \mathrm{D}$ point cloud is obtained directly from the integration of laser scanner and positioning and orientation sensors. Moreover, LiDAR data is not influenced by imaging conditions.

During the past decade, several methods using airborne LiDAR data for building change detection have been developed. They can be divided into two categories: change detection using multi-temporal LiDAR data or combining LiDAR data with another data source. In the first category, the building changes are usually identified by comparing interpolated digital surface models (DSM) from two different epochs (Choi et al., 2009; Murakami et al., 1999; Pang et al., 2014; Pirasteh et al., 2019; Teo and Shih, 2013; Tuong Thuy Vu et al., 2004; Vogtle and Steinle, 2004). According to Butkiewicz et al. (2008), an alternative to avoid DSM interpolation errors is to perform the changes detection directly over the LiDAR point cloud, i.e., irregularly spaced LiDAR points. In the second category, the LiDAR data can be used to update an existing geographic database or combined with different data sources for change detection. In Awrangjeb et al. (2015) the authors proposed a method for updating building information in a topographic map using LiDAR point cloud data. In Huang and Chen (2007), Malpica and Alonso (2010) and Matikainen et al. (2010), the detection process is based on the comparison of an existing building map (first epoch) with airborne LiDAR and aerial image data (second epoch). Malpica et al. (2013) uses satellite image and LiDAR data to update a vector geospatial database. In Du et al. (2016) is proposed an automatic method using old aerial images and LiDAR data. Zhou et al. (2018) provides a novel method for change detection between past LiDAR and new images.

In the context of building change detection from LiDAR data, differentiating between the building and non-building changes is a challenge, since the classification depends on some factors,

* Corresponding author 
such as object features and point cloud density. To overcome this problem, some works explored the semantic information derived from images (Huang and Chen, 2007; Malpica et al., 2013; Malpica and Alonso, 2010; Matikainen et al., 2010). In a real scenario, LiDAR and image integration makes the acquisition process more expensive, being impractical for some emerging countries. In this sense, some authors have explored the geometry information derived from the LiDAR point cloud. In Vogtle and Steinle (2004), the change clusters are classified using a set of attributes: the number of significant border gradients, height texture, first/last pulse differences, shape parameters and laser pulse intensities. In Choi et al. (2009), the clusters are classified as ground, vegetation or building; being considered roughness, height and size as attributes. In the paper of Teo and Shih (2013) the change clusters are divided into building and vegetation considering the surface roughness attribute, which is estimated using height gradient variance. In Pang et al. (2014), the random sample consensus (RANSAC) fitting algorithm is used to distinguish the true changed buildings from trees. In Awrangjeb et al. (2015) planar roof segments are extracted over change areas, being the vegetation clusters eliminated using some information such as area, and orientation. In Du et al. (2016), the non-building changes are removed considering two strategies: the normal variance direction of LiDAR points is used to remove vegetated areas for positive building changes (newly building or taller) and nEGI (normalized Excessive Green Index) is used for negative building changes (demolish building or lower). In some works, the area threshold is applied to eliminate small changes, which are usually related to non-building changes. In Pirasteh et al. (2019), for instance, it is considered that the smallest building has $75 \mathrm{~m}^{2}$, eliminating most of the vegetation areas. Thus, a negative aspect is that changes in buildings with an area smaller than this threshold are not identified.

In this paper, a building change detection using multi-temporal airborne LiDAR data is proposed. The main contribution of this approach consists in the use of the Shannon entropy concept as a metric to distinguish the building and vegetation change clusters. In the proposed method, the change detection is obtained subtracting the interpolated DSM from two different epochs, obtaining a differential DSM (dDSM). Then, a height entropy value is determined for each change candidate cluster, which is estimated directly over the original LiDAR to preserve the multiple return geometry.

The remainder of this paper is organized as follows. The proposed method is described in Section 2. The study area and dataset are presented in Section 3. The experiments and discussion are presented in Section 4. Finally, conclusions and further investigation are summarized in Section 5.

\section{METHOD}

The proposed method is composed of three main steps: preprocessing, change detection and building change detection, as highlighted in the flowchart shown in Figure 1. The input data are two LiDAR point clouds of two epochs ( $t_{1}$ and $t_{2}$ ), whereas the output corresponds to a building change map. The building changes clusters are divided into two classes: constructed or demolished buildings. In Figure 1 the contribution of the proposed method is highlighted in blue dotted lines. It is assumed that both LiDAR data are referenced to the same global reference system.

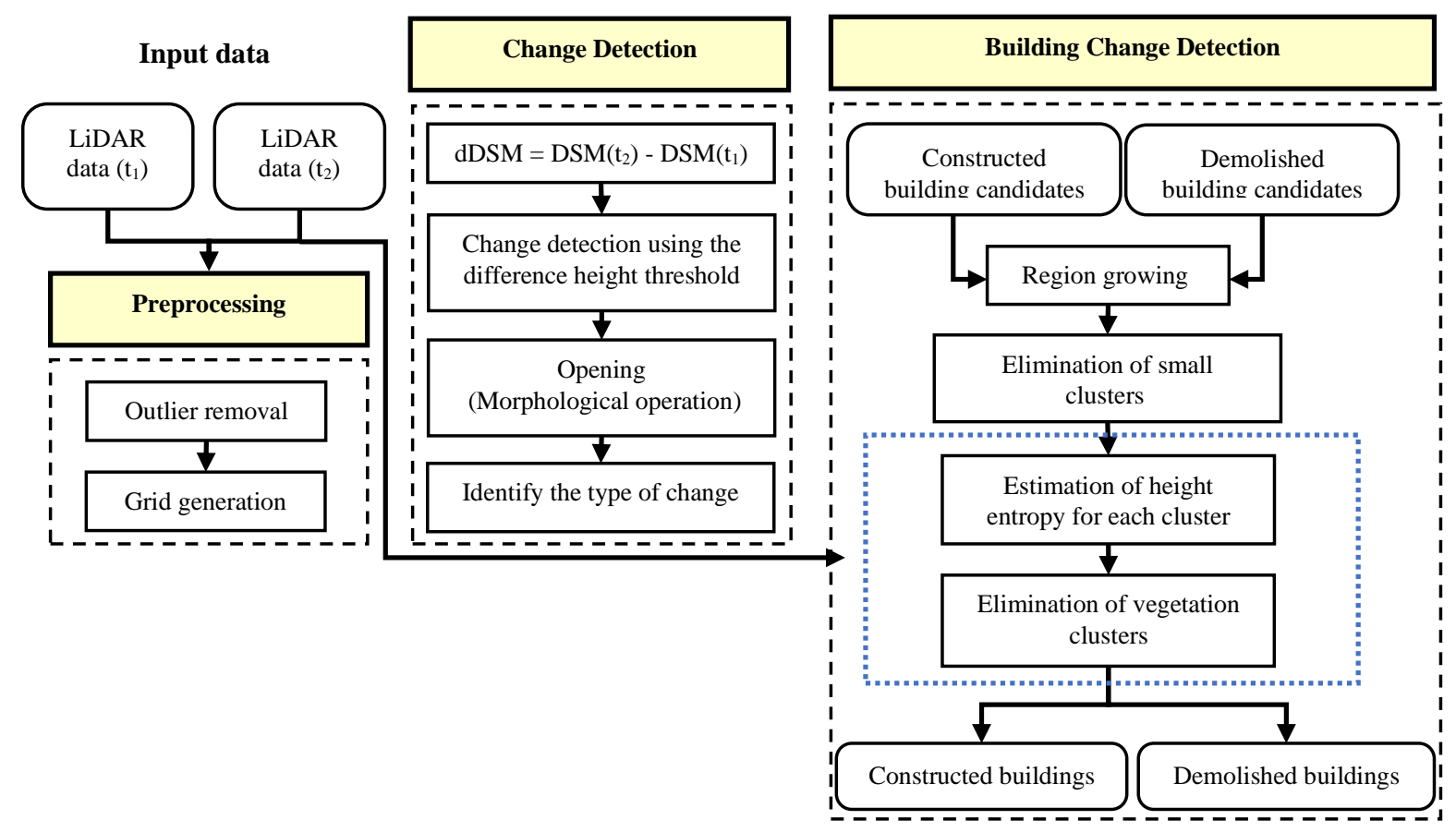

Figure 1. Building change detection method.

\subsection{Preprocessing}

The LiDAR point cloud available may contain outlying points that should be excluded before the data processing (Ben-Gal, 2005). In this paper, the method proposed by Carrilho et al. (2018), cell histogram filter ( $\mathrm{CH}$ filter), was considered. The method consists of identifying the outlying points by means of the analysis of the height frequency histogram generated locally. In this context, a frequency threshold $\left(T_{f}\right)$ should be adopted. 
After the outlier removal from both LiDAR point clouds, acquired in epochs $t_{l}$ and $t_{2}$, the grid data, i.e., the DSM can be generated. The DSM generation was performed using the lasgrid tool of LAStools software (http://rapidlasso.com/lastools/). In the LiDAR point cloud, the multiple returns can be affected by scan pulse, flight planning and scene characteristics (vegetation leaf cover), which hardly remain constant in independent surveys at two different epochs. Thus, to avoid false changes derived from multiple return variations, only the first return was considered.

\subsection{Change detection}

After the preprocessing step, the difference DSM (dDSM) is obtained employing Equation 1. The dDSM corresponds to a height difference map, which allows locating the potential change areas. To eliminate the small height variations a height threshold $\left(T_{H}\right)$ is applied, similar to Teo and Shih (2013). In this case, the pixels with absolute values larger than the height threshold are labeled as potential changes, otherwise, they are considered as no changes. Figure 2a presents an example of potential changes.

$$
d D S M=D S M\left(t_{2}\right)-D S M\left(t_{1}\right)
$$

where $\operatorname{DSM}(t)$ is the DSM at epoch $t$, and $d D S M$ is the height difference between two epochs.

The dDSM usually has noise problems (Figure 2a), which are related to grid interpolation errors. This problem normally occurs in the regions near the steep edge and can be minimized by applying morphological filters, specifically the opening operator in this case, which is defined by an erosion followed by a dilation. In Figure $2 b$ is presented the result after applying the opening operator considering a disk as structuring element.

In the sequence, the pixels labeled as potential changes, i.e., only those that $|\mathrm{dDSM}(\mathrm{i}, \mathrm{j})|>T_{H}$, are separated into two classes: constructed and demolished building candidates. This classification, as shown in Figure 2c, is performed by analysing the signal of height variation, i.e., whether the height variation is positive $(\operatorname{dDSM}(i, j)>0)$, then pixel is labelled as constructed building candidate, otherwise the pixel is labelled as demolished candidate $(\operatorname{dDSM}(\mathrm{i}, \mathrm{j})<0)$.

To obtain the clusters related to each change, the region growing was performed considering the pixels connectivity criterion, being used 8-connected neighbourhood. In this work, the segmentation process was applied to each class separately, avoiding the grouping of different types of changes in the same cluster. After this process, it is possible to determine the area of each cluster. Considering that the small changes are usually related to commission errors, an eliminating process can be performed using an area threshold $\left(T_{A}\right)$. Figure $2 \mathrm{~d}$ shows the result after eliminating small areas.

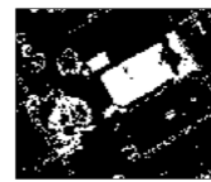

(a)

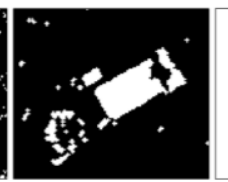

(b)

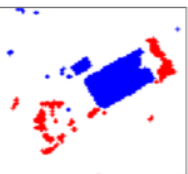

(c)

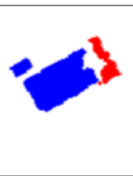

(d)
Figure 2. Change detection using the height threshold (a).

Result after applying the opening operator (b). Classification of pixel changes into constructed (blue) and demolished building

(red) (c). Result after eliminating the small clusters (d).

\subsection{Building change detection using the entropy concept}

As pointed by Murakami et al. (1999) and Teo and Shih (2013), the building change detection methods based on height difference may present commission errors, normally related to vegetation areas. An alternative to mitigate this problem is the application of the Shannon entropy concept, which is a measure of dispersion in a given domain (neighbourhood). Considering the geometric characteristics, it is expected that the vegetation presents a high magnitude of entropy, whereas the building has a smaller magnitude. In this work, the height entropy of a given point is estimated using Equation 2 (Oliveira and Galo, 2017), which corresponds to an adaptation of the Demantké et al. (2011).

$$
E_{j}=n^{-1} \Sigma\left[-\left(h_{i}-h_{\min }\right) \ln \left(h_{i}-h_{\min }\right)\right] \quad \forall h_{i \neq} h_{\min } \quad i=1 \ldots n
$$

In Equation 2, the height entropy value $\left(E_{j}\right)$ is computed considering a neighbourhood defined by a vertical cylinder of radius $R$ centered on each point of interest. The $h_{i}$ represents the height of generic point $i$, whereas $h_{\min }$ corresponds to the minimum height in its neighbourhood.

The height entropy is directly computed over the LiDAR point cloud, i.e., with all laser returns. The main advantage is related to the conservation of multiple return geometry, especially in vegetation areas. In general, the height entropy estimation is divided into two mains stages. In the first, for each change pixel, it is determined the closest point in the LiDAR point cloud. In the second, the height entropy value is computed considering all points inside each vertical cylinder. The estimated height entropy for the point is associated with the corresponding change pixel.

The height entropy was estimated for both $t_{1}$ and $t_{2}$ LiDAR point clouds. In this context, the pixel change that presents $|\operatorname{dDSM}(\mathrm{i}, \mathrm{j})|>T_{H}$ and $\operatorname{dDSM}(\mathrm{i}, \mathrm{j})<0$, i.e., demolished building candidate, has its height entropy value estimated based on $t_{1}$ LiDAR point cloud, whereas for the pixel that presents $|\operatorname{dDSM}(\mathrm{i}, \mathrm{j})|>T_{H}$ and $\operatorname{dDSM}(\mathrm{i}, \mathrm{j})>0$, i.e., constructed building candidate, has its height entropy value estimated based on $t_{2}$ LiDAR point cloud. This strategy was adopted considering the idea that a demolished building exists in epoch 1 and does not exist in epoch 2, whereas the constructed building does not exist in epoch 1 and exists in epoch 2. In Figure 3 is presented one example of height entropy estimation for a pixel classified as a demolished building candidate. The same process is used for constructed building candidates, however, in this case, the LiDAR data at epoch $t_{2}$ is considered.

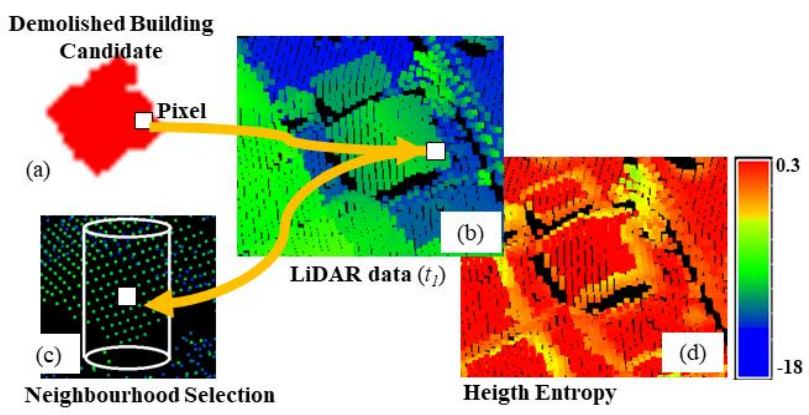

Figure 3. Estimation of the height entropy. Cluster related to demolished area (a). LiDAR point cloud in the epoch $t_{l}(\mathrm{~b})$. Neighbourhood selection using a vertical cylinder (c). Height entropy map (d). 


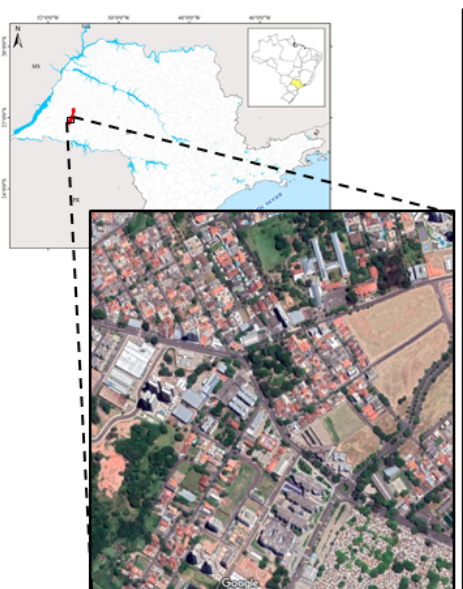

(a)

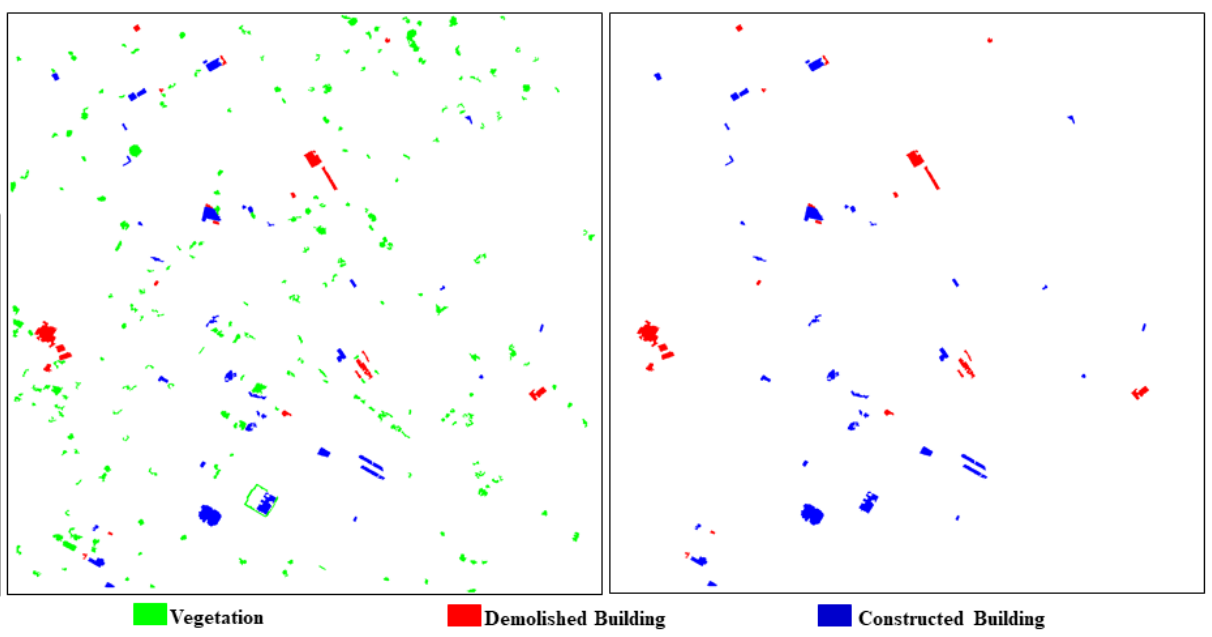

(b)

(c)

Figure 4. Study area in Presidente Prudente/Brazil (a). Results of proposed method (b-c). Vegetation detection using the entropy concept (b). Building changes (c).

For each change cluster, a unique entropy value is determined based on the entropy value of each pixel. At this stage, several alternatives could be applied, such as the mean, the median, maximum, minimum, etc. In our work, median value $\left(E_{m e d}\right)$ is adopted as a cluster attribute. The median was selected to minimize the influence of outlier values, i.e., height entropy values that differ from the central point. This is a recurring situation in roof building areas since the roof surface has a low entropy magnitude and the edge has a high magnitude. To verify whether a given cluster corresponds to a building or vegetation change, the magnitude of entropy value $\left(E_{m e d}\right)$ is compared to an entropy threshold $\left(T_{E}\right)$. Whether the magnitude of $E_{\text {med }}$ is smaller than the threshold, then the cluster is labeled as building change, otherwise, the cluster is considered as a vegetation change.

\section{STUDY AREA AND DATASET}

\subsection{Study area}

The study area, located in Presidente Prudente city, in the Southeast region of Brazil, has an area of approximately $1 \mathrm{~km}^{2}$ $(1 \mathrm{~km} \mathrm{x} 1 \mathrm{~km})$. It is composed of different types of objects, such as buildings, trees, and streets. In Figure $4 \mathrm{a}$ is presented the geographic location and an aerial image of the interest area. In this area, there are buildings with different shapes, heights, and dimensions. In the upper-left corner, there is a high concentration of residential houses (with one or two floors), whereas in the lower and central parts, it is possible to observe taller and broader buildings.

\subsection{Dataset}

The experiments were performed considering the point cloud data of two epochs (2012 and 2014). Both datasets were acquired by an airborne RIEGL LMS-Q680i system from the Sensormap Company. This system has a precision of up to $2 \mathrm{~cm}$ in the range measurement, a LASER pulse repetition rate of up to $400 \mathrm{kHz}$ and an measurement rate of up to $266 \mathrm{kHz}$ at a $60^{\circ}$ scan angle. The system can also store multiple returns. In Table 1 are presented some information about each aerial acquisition mission.

\begin{tabular}{ccc}
\hline Dataset & $\mathbf{2 0 1 2}$ & $\mathbf{2 0 1 4}$ \\
\hline Flying height & $900 \mathrm{~m}$ & $900 \mathrm{~m}$ \\
Average point spacing & $0.44 \mathrm{~m}$ & $0.44 \mathrm{~m}$ \\
Average point density & 5.1 points $/ \mathrm{m}^{2}$ & $5.2 \mathrm{points} / \mathrm{m}^{2}$ \\
Total number of points & $3,125,730$ & $3,207,329$ \\
\hline
\end{tabular}

Table 1. Information related to each data.

\section{RESULTS AND DISCUSSION}

\subsection{Results}

As described in Section 2, some parameters must be considered to apply the proposed method. In Table 2 is presented the value and description related to each parameter. The parameters include the grid cell size, the height difference threshold, the structuring element of the morphological filter, the radius of the cylinder, the area threshold, and the entropy threshold. The values of the thresholds were defined empirically.

The results of the proposed method are presented in Figure 4. In Figure $4 \mathrm{~b}$ is presented all potential clusters related to changes with an area bigger than $T_{A}$. In this case, the changes are divided into three classes: vegetation, demolished or constructed building. Figure $4 \mathrm{c}$ shows just demolished and constructed buildings.

\begin{tabular}{|c|c|c|}
\hline Parameters & Values & Description \\
\hline Grid cell size & $0.5 \mathrm{~m}$ & $\begin{array}{l}\text { Grid cell size used to generate } \\
\text { the DSM. }\end{array}$ \\
\hline $\begin{array}{l}\text { Height threshold } \\
\left(T_{H}\right)\end{array}$ & $2 \mathrm{~m}$ & $\begin{array}{l}\text { To verify if a given pixel } \\
\text { correspond to a change. }\end{array}$ \\
\hline $\begin{array}{c}\text { Structuring } \\
\text { element }\end{array}$ & $\begin{array}{l}\text { Disk of } \\
\text { radius } \\
1 \mathrm{~m}\end{array}$ & $\begin{array}{l}\text { Used to perform the } \\
\text { morphological operation } \\
\text { (opening). }\end{array}$ \\
\hline $\begin{array}{l}\text { Radius of the } \\
\text { cylinder }(R)\end{array}$ & $1 \mathrm{~m}$ & $\begin{array}{l}\text { Used in the neighbourhood } \\
\text { selection. }\end{array}$ \\
\hline $\begin{array}{c}\text { Area threshold } \\
\left(T_{A}\right)\end{array}$ & $20 \mathrm{~m}^{2}$ & To remove small objects. \\
\hline $\begin{array}{l}\text { Entropy threshold } \\
\left(T_{E}\right)\end{array}$ & 2 & To identify tree clusters. \\
\hline
\end{tabular}

Table 2. Change detection parameters.

To perform the quantitative analysis, the following quality parameters, as described in Wiedemann et al. (1998) and Sokolova et al. (2006), were computed: completeness (Comp), correctness (Corr), accuracy $(A c c u)$ and $F_{\text {score }}$. Each change 
cluster was manually verified through a visual inspection using aerial images and LiDAR data from both epochs. In Table 2 is presented the quality parameters estimated for the study area, as well as the average value of each metric.

\begin{tabular}{ccccc}
\hline Classes & $\begin{array}{c}\text { Comp. } \\
(\boldsymbol{\%})\end{array}$ & $\begin{array}{c}\text { Corr. } \\
(\boldsymbol{\%})\end{array}$ & $\begin{array}{c}\text { Accu. } \\
(\boldsymbol{\%})\end{array}$ & $\begin{array}{c}\boldsymbol{F}_{\text {score }} \\
(\boldsymbol{\%})\end{array}$ \\
\hline Constructed Building & 100 & 73.2 & 85.8 & 84.5 \\
Demolished Building & 94.7 & 69.2 & 94.9 & 80.0 \\
\hline Mean & 97.3 & 71.2 & 90.4 & 82.3 \\
\hline
\end{tabular}

Table 3. Quality parameters.

In Figure 5 is highlighted some change areas obtained by the proposed method. To facilitate the visual analysis, it is also presented the corresponding aerial images and DSMs to each epoch. Figure 6 presents some change areas that were incorrectly classified as building changes. In this case, it is presented the DSMs and height profiles.

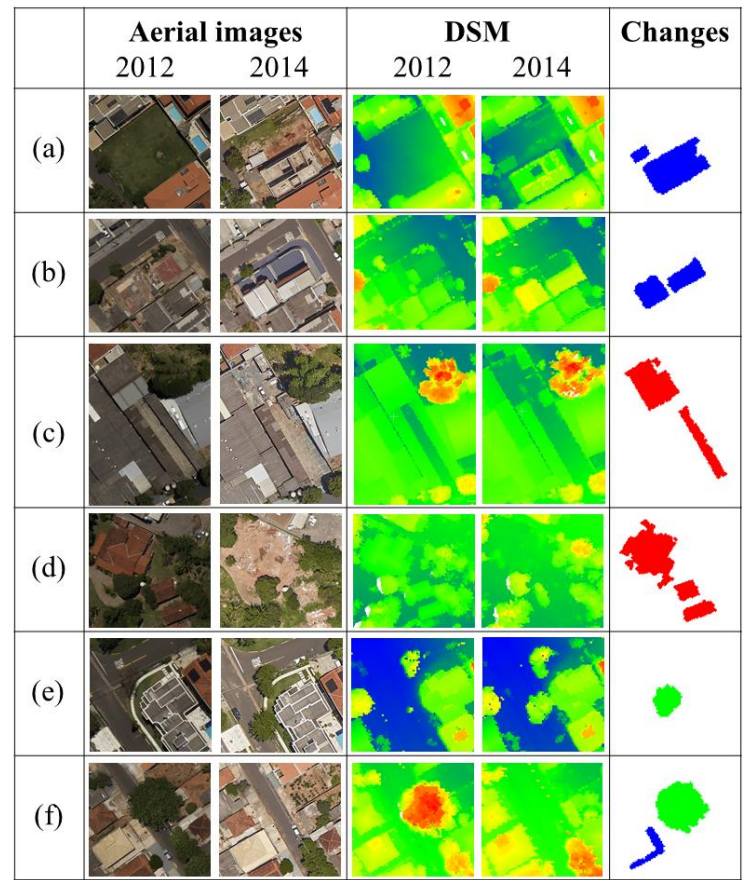

Figure 5. Change areas identified using the proposed method. Constructed buildings (blue, a, b and f), demolished buildings (red, c and d), and vegetation changes (green, e and f).

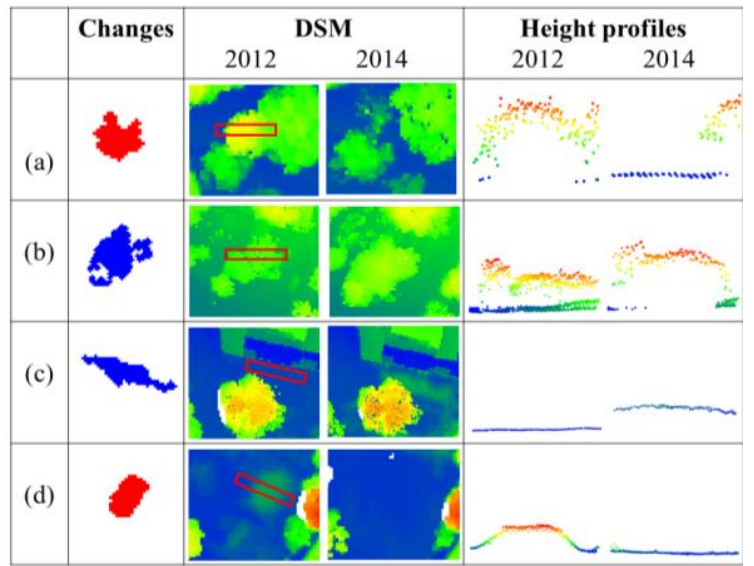

Figure 6. Change areas incorrectly classified as building changes. Vegetation $(a, b)$ and ground $(c, d)$ changes.

\subsection{Results discussion}

From visual analysis (Figure 4), it is possible to observe that a large number of clusters of vegetation changes were identified. Since the focus of this work is to find building changes, the identification and elimination of vegetation changes allow to reduce the commission errors, i.e., false negatives. In Figures 5a-5d are presented constructed and demolished buildings, whereas in Figures $5 \mathrm{e}$ and $5 \mathrm{f}$ are presented two vegetation changes correctly identified. In Figure $5 f$ is also shown a small building change, which is related to a building extension.

Analyzing the completeness metric (Table 3), it is possible to notice that constructed and demolished building classes reached $100 \%$ and $94.7 \%$, respectively. This indicates that most of the building changes were identified, i.e., the proposed method had a small omission error rate. This is an important achievement since the verification process of omission errors is much more costly and labor-intensive than the commission errors (Murakami et al., 1999).

Considering the correctness metric (Table 3), the constructed building class presented value around $73 \%$ against $69 \%$ of the demolished building class, indicating a low commission error rate. This result is directly related to building change detection step, where the building change candidates were separated into building and vegetation changes through height entropy concept.

The proposed method presented average completeness and correctness around $97 \%$ and $71 \%$, respectively, being considered building changes larger than $20 \mathrm{~m}^{2}$. The method proposed by Teo and Shih (2013) presented correctness around $80 \%$ for building changes larger than $50 \mathrm{~m}^{2}$. In Pang et al. (2014), the authors also considered building changes larger than $50 \mathrm{~m}^{2}$, the completeness and correctness were around $98 \%$ and $91 \%$, respectively. Matikainen et al. (2010) perform a quality analysis considering building changes larger than $20 \mathrm{~m}^{2}$. The constructed building class presented completeness and correctness values around $69 \%$ and $56 \%$, respectively, whereas the demolished class has completeness and correctness around $43 \%$ and $68 \%$, respectively. This comparison indicates that the results obtained by the proposed method are compatible with the previously developed methods, presenting a high potential in detecting building changes larger than $20 \mathrm{~m}^{2}$.

In Figure 6 are highlighted some commission errors, which are corresponding to vegetation (Figures $6 \mathrm{a}$ and $6 \mathrm{~b}$ ) and ground changes (Figures $6 \mathrm{c}$ and $6 \mathrm{~d}$ ). This type of error is directly related to the estimated height entropy value for each cluster and the entropy threshold $\left(T_{E}\right)$ adopted. In some cases, the height entropy estimated for the cluster may have a similar value to buildings, as the laser pulse might fail to penetrate the tree canopy due to high leaf density. In the case of ground, commission errors were already expected, since the ground has similar behavior to the roof building, i.e., locally flat surface.

In summary, the qualitative and quantitative analysis indicates that the proposed method has the potential to be used to identify building changes using LiDAR data from two epochs. In addition, the results showed the potential of the adopted strategy to minimize commission errors, usually related to vegetation changes. 


\section{CONCLUSION}

This paper proposes a method for building change detection using LiDAR data. The main contribution is related to the use of height entropy concept to classify the change areas into building or vegetation. This method was robust in detecting building changes, having also the potential to identify small changes (larger than $20 \mathrm{~m}^{2}$ ). The drawback of the proposed method is directly related to the definition of the entropy threshold, which was empirically defined for the dataset used in the experiments.

As for future research, it is suggested to apply a technique that allows the automatic definition of the entropy threshold. Besides, it is interesting to explore other forms of calculating the entropy.

\section{ACKNOWLEDGEMENTS}

The authors would like to thank Graduate Program on Cartographic Sciences from FCT-UNESP, Presidente Prudente-SP/Brazil; Sensormap Geotecnologia for providing the LiDAR data; São Paulo Research Foundation - FAPESP (grant 2019/05268-8) for supporting this research; National Council for Scientific and Technological Development - CNPq (grant 304189/2016-2); and Coordenação de Aperfeiçoamento de Pessoal de Nível Superior - CAPES (Finance Code 001).

\section{REFERENCES}

Awrangjeb, M., Fraser, C. S., Lu, G., 2015. Building change detection from LiDAR point cloud data based on connected component analysis. ISPRS Ann. Photogramm. Remote Sens. Spatial Inf. Sci., II-3/W5, 393-400. https://doi.org/10.5194/isprsannals-II-3-W5-393-2015

Ben-Gal, I., 2005. Outlier detection. Data Mining and Knowledge Discovery Handbook. Springer, pp. 131-146.

Butkiewicz, T., Chang, R., Wartell, Z., Ribarsky, W., 2008. Visual analysis and semantic exploration of urban LiDAR change detection. Computer Graphics Forum 27, 903-910. https://doi.org/10.1111/j.1467-8659.2008.01223.x

Carrilho, A. C., Galo, M., Santos, R. C., 2018. Statistical outlier detection method for airborne LiDAR data. Int. Arch. Photogramm. Remote Sens. Spatial Inf. Sci., XLII-1, 87-92. https://doi.org/10.5194/isprs-archives-XLII-1-87-2018

Choi, K., Lee, I., Kim, S., 2009. A feature based approach to automatic change detection from LIDAR data in urban areas. Laser scanning 09. Vol XXXVIII Part 3/W8, on CDROM.

Demantké, J., Mallet, C., David, N., Vallet, B., 2011. Dimensionality based scale selection in 3D lidar point clouds. Int. Arch. Photogramm. Remote Sens. Spat. Inf. Sci, 38, W12.

Du, S., Zhang, Y., Qin, R., Yang, Z., Zou, Z., Tang, Y., Fan, C., 2016. Building change detection using old aerial images and new LiDAR data. Remote Sensing 8, 1030. https://doi.org/10.3390/rs8121030

Huang, C., Chen, L., 2007. Detection of building changes from LiDAR data and aerial imagery. ACRS 2007: Proceedings of the 28th Asian Conference on Remote Sensing. pp. 12-16.

Malpica, J. A., Alonso, M. C., 2010. Urban changes with satellite imagery and LiDAR data. Int. Arch. Photogramm. Remote Sens. Spatial Inf. Sci., XXXVIII, 853-858.

Malpica, J. A., Alonso, M. C., Papí, F., Arozarena, A., Martínez De Agirre, A., 2013. Change detection of buildings from satellite imagery and lidar data. International Journal of Remote Sensing 34, 1652-1675. https://doi.org/10.1080/01431161.2012.725483

Manno-Kovacs, A., Sziranyi, T., 2015. Orientation-selective building detection in aerial images. ISPRS Journal of Photogrammetry and Remote Sensing 108, 94-112. https://doi.org/10.1016/j.isprsjprs.2015.06.007

Matikainen, L., Hyyppä, J., Ahokas, E., Markelin, L., Kaartinen, H., 2010. Automatic detection of buildings and changes in buildings for updating of maps. Remote Sensing 2, 1217-1248. https://doi.org/10.3390/rs2051217

Murakami, H., Nakagawa, K., Hasegawa, H., Shibata, T., Iwanami, E., 1999. Change detection of buildings using an airborne laser scanner. ISPRS Journal of Photogrammetry and Remote Sensing 54, 148-152. https://doi.org/10.1016/S09242716(99)00006-4

Oliveira, R. A. R., Galo, M., 2017. Classificação de feições na superfície a partir de dados LiDAR e medidas de entropia e desvio padrão das altitudes. Proceedings of the XVIII Simpósio Brasileiro de Sensoriamento Remoto. Santos, pp. 2892-2899.

Pang, S., Hu, X., Wang, Z., Lu, Y., 2014. Object-Based analysis of airborne LiDAR data for building change detection. Remote Sensing 6, 10733-10749. https://doi.org/10.3390/rs61110733

Pirasteh, S., Rashidi, P., Rastiveis, H., Huang, S., Zhu, Q., Liu, G., Li, Y., Li, J., Seydipour, E., 2019. Developing an algorithm for buildings extraction and determining changes from airborne LiDAR, and comparing with R-CNN method from drone images. Remote Sensing 11, 1272. https://doi.org/10.3390/rs11111272

Sokolova, M., Japkowicz, N., Szpakowicz, S., 2006. Beyond Accuracy, F-Score and ROC: A Family of Discriminant Measures for Performance Evaluation. AI 2006: Advances in Artificial Intelligence. Springer Berlin Heidelberg, Berlin, Heidelberg, $\quad$ pp. https://doi.org/10.1007/11941439_114

1015-1021.

Teo, T.-A., Shih, T.-Y., 2013. Lidar-based change detection and change-type determination in urban areas. International Journal of Remote Sensing 34, 968-981. https://doi.org/10.1080/01431161.2012.714504

Tuong Thuy Vu, Matsuoka, M., Yamazaki, F., 2004. LIDARbased change detection of buildings in dense urban areas. IEEE International Geoscience and Remote Sensing Symposium, 2004. IGARSS '04. Proceedings. 2004. Anchorage, AK, USA, pp. 3413-3416. https://doi.org/10.1109/IGARSS.2004.1370438

United Nations, 2019. Sustainable Development Goals. https://www.un.org/sustainabledevelopment/sustainabledevelopment-goals/ (28 October 2019).

Vogtle, T., Steinle, E., 2004. Detection and recognition of changes in building geometry derived from multitemporal laserscanning data. IAPRS 428-433.

Wiedemann, C., Heipke, C., Mayer, H., Jamet, O., 1998. Empirical evaluation of automatically extracted road axes. Empirical Evaluation Techniques in Computer Vision. Citeseer, pp. $172-187$.

Zhou, K., Gorte, B., Lindenbergh, R., Widyaningrum, E., 2018. 3D Building change detection between current VHR images and past LiDAR data. Int. Arch. Photogramm. Remote Sens. Spatial Inf. Sci., XLII-2, 1229-1235. https://doi.org/10.5194/isprsarchives-XLII-2-1229-2018 